\title{
High-Performance Fingerprint Localization in Massive MIMO System
}

\author{
Yijie REN, Zhixing XIAO, Yuan TANG, Fei TANG, Xiaojun WANG ${ }^{1}$ and Xiaoshu \\ CHEN \\ School of Information Science and Engineering, National Mobile Communications \\ Research Laboratory, Southeast University
}

\begin{abstract}
Location-based service (LBS) for both security and commercial use is becoming more and more important with the rise of $5 \mathrm{G}$. Fingerprint localization $(\mathrm{FL})$ is one of the most efficient positioning methods for both indoor and outdoor localization. However, the positioning time of previous research cannot achieve real-time requirement and the positioning error is meter level. In this paper, we concentrated on high-performance in massive multiple-in-multiple-out (MIMO) systems. Principal Component Analysis (PCA) is applied to reduce the dimension of fingerprint, so that the positioning time is about tens of milliseconds with lower storage. What's more, a novel fingerprint called Angle Delay Fingerprint (ADF) is proposed. Simulation result of the positioning method based on ADF shows the positioning error is about 0.3 meter and the positioning time is about hundreds of milliseconds, which is much better than other previous known methods. ${ }^{2}$
\end{abstract}

Keywords. Fingerprint localization, High-performance, Data dimension reduction, Massive MIMO

\section{Introduction}

Recently, with the rise of 5th Generation Mobile Communication Technology (5G), the characteristics such as high speed, low latency, large bandwidth and massive Internet of things make people enter the age of the Internet of everything fast. Whether it is autodriving or smart home, or emergency rescue, they all rely on location base service deeply. However, tall buildings and trees rise up in the city, which makes the propagation environment of wireless signals complicated and changeable. Thus, high positioning performance of mobile terminals is required.

The traditionally employed positioning methods can be divided into two kinds. One is satellite positioning, such as Global Positioning System (GPS) and Big Dipper. The other is based on the received signal strength (RSS), the time difference of arrival (TDOA), angel of arrival (AOA) which assumes the wireless signal transmit along the line-of-sight (LOS) path [1-3]. However, the classic location techniques mentioned above are sensitive to surrounding environments and cannot perform well in dense outdoor circumstances.

\footnotetext{
${ }^{1}$ Corresponding author: Xiaojun WANG, No. 2, Southeast University Road, Nanjing, China; E-mail: wxj@seu.edu.cn.

${ }^{2}$ Foundation items: Social Development Projects of Jiangsu Science and Technology Department (No.BE2018704).
} 
Compared with the traditional positioning methods, fingerprint localization is simple and efficient. It uses multipath information efficiently. The idea of fingerprint localization is that the propagation of wireless signal is strongly dependent on wireless environment, so that the positioning problem can be thought as a pattern recognition issue [4-7]. Fingerprint scheme can be divided into two phases. In offline phase, a database including fingerprints of reference points in target localization area is established. In online phase, we extract fingerprints and match them with the fingerprints in the database to get the estimated location.

There are two types of fingerprint. The first one is receiving signal strength information measured from access points (APs), which is simple and cost-effective. It is usually used in indoor positioning. The second type of parameters is multipath characteristics from wireless channels, including angel of arrival (AOA) [8], channel state information (CSI) [9], power delay profile (PDP) [10].

Massive multiple-in-multiple-out (MIMO) [11] is considered as one of the leading technologies of $5 \mathrm{G}$, which can provide more multipath information for fingerprint localization. Traditional CSI fingerprint is extracted from time domain. Due to the application of massive MIMO, the information of time domain can be transformed into angle-delay domain, which improve the stability of fingerprint localization [12]. Angel delay Channel Power Matrix (ADCPM) is a kind of fingerprint based on massive MIMO system [13]. A deep learning approach based on ADCPM and a fast fingerprint localization method based on ADCPM were proposed in [14]. These researches based on ADCPM improve the performance of fingerprint positioning to some extent, but they are also limited.

In this paper, we focus on high-performance fingerprint localization problem in massive MIMO system. First, Principal Component Analysis (PCA) is used to reduce the dimension of ADCPM, which accelerates the online positioning process and reduces storage overhead. Second, Angle Delay Fingerprint (ADF) is proposed based on ADCPM. The average positioning error is about one-third of ADCPM. The average positioning time is about tens of milliseconds. The positioning performance is much better than previous methods based on ADCPM. Finally, many simulations are conducted to demonstrate the performance of the two new fingerprints.

The rest of the paper is organized as follow. In Section 2, the system model and fingerprint extraction method is stated. The compressed fingerprint localization method based on PCA and the positioning method based on ADF are presented in section 3. In section 4 , simulation results are provided to demonstrate the performance of the proposed localization methods.

\section{Fingerprint Extraction}

In this section, the channel model and fingerprint extraction in the Angle-Delay domain are presented. The base station (BS) is equipped with $N_{t}$ antennas in the form of uniform linear array (ULA), and by uplink channel estimation at the base station, CSI is considered to be known.

\subsection{Channel Model}

We assume that users are randomly distributed in the localization area, which is simple and without loss of generality. The channel model is the same as [13]. Firstly, the 
Channel Impulse Response (CIR) of each user is given, where the multipath characteristic is included. To combine the AOA information, the array response vector corresponding to the AOA is considered. The scatters are independent and uniform in the target area. Then, the overall channel frequency response (CFR) of each user is generated by discrete Fourier transform (DFT). Detailed derivations are presented in [13].

\subsection{Angle-delay Fingerprint Extraction}

The fingerprint used for positioning is extracted from massive MIMO system, which indicates much more multipath information in both angle and delay domain. Using the CFR generated from previous section, the angle-delay fingerprint is easily extracted. By multiplying the DFT and unitary DFT matrix on the left and right, the CFR matrix is mapped from the frequency domain to the angle-delay domain, which is called angledelay domain channel response matrix (ADCRM). Finally, in order to get more visualized data, we use the expectation of the Hadamard product of two ADCRM as the angle-delay channel power matrix (ADCPM). The derivation procedure and proof of ADCPM property is omitted, which can be referred to [13] for details.

\section{Proposed Fast Fingerprint Localization Algorithms}

In this section, two new fingerprint localization algorithms will be proposed. The process of fingerprint positioning includes online phase and offline phase. The overall process is shown as Figure.1.

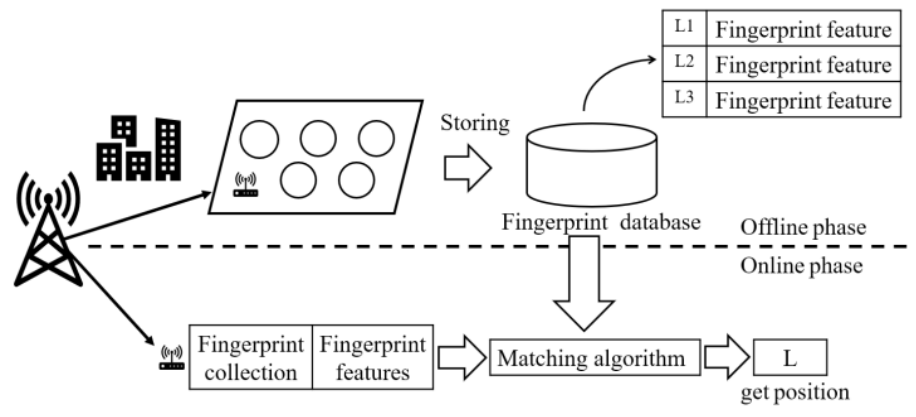

Figure 1. The process of fingerprint localization.

After establishing a fingerprint database in offline phase, the fingerprints extracted from unknown points to be positioned are matched with the fingerprints in database in online phase. Different fingerprint similarity criteria are available.

In this paper, we use Center Angle of Arrival (CAOA) clustering algorithm for data preprocessing, and use Weighted K Nearest Neighbor (WKNN) as the basic matching algorithm [13]. The similarity of two fingerprints is measured using the Manhattan Distance (MD) criterion which is shown in the following formula:

$$
\operatorname{dist}_{\text {man }}\left(\boldsymbol{x}_{i}, \boldsymbol{x}_{j}\right)=\left\|\boldsymbol{x}_{i}-\boldsymbol{x}_{j}\right\|_{1}=\sum_{u=1}^{n}\left|x_{i u}-x_{j u}\right|
$$


In the formula, $\mathrm{N}$ is the dimension of the fingerprint, $x_{i}$ and $x_{j}$ are two $\mathrm{n}$-dimensional vectors. The similarity of two fingerprints is higher, as the value of $\operatorname{dist}_{\text {man }}\left(x_{i}, x_{j}\right)$ is smaller and vice-versa.

\subsection{The Compressed Fingerprint Localization Method Based on PCA}

ADCPM is a high-dimensional data. Using ADCPM in fingerprint positioning will bring high storage overhead and high computation cost in online matching phase. Data dimension reduction can extracted efficient information from original data.

PCA is a widely used method for data dimension reduction. The main idea of PCA is that the original data can be reconstructed in feature space by eigenvalue decomposition. Thus, the $\mathrm{n}$-dimensional data can be represented by $\mathrm{k}$-dimensional eigenvalue. $\mathrm{N}$ is greater than $\mathrm{k}$, so the original data can be compressed. The flowchart of PCA algorithm is shown in Table 1.

Table 1. The Flowchart of PCA Algorithm

\begin{tabular}{|l|}
\hline 1: Input: Original data $\left\{x_{1}, x_{2}, \ldots, x_{m}\right\}$, Low dimensional spatial dimensions $d^{\prime} ;$ \\
\hline 2: Centralize all samples: $x_{i} \leftarrow x_{i}-\frac{1}{m} \sum_{i=1}^{m} x_{i} ;$ \\
\hline 3: Calculate the sample covariance matrix $X X^{T} ;$ \\
\hline 4: Decompose the covariance matrix $X X^{T}$ by eigenvalue; \\
\hline 5: Pick the eigenvectors corresponding to the largest eigenvalues $w_{1}, w_{2}, \ldots, w_{d^{\prime}} ;$ \\
\hline 6: Output: The basic vectors of reconstructed plane $\mathrm{W}=\left(w_{1}, w_{2}, \ldots, w_{d^{\prime}}\right)$. \\
\hline
\end{tabular}

After extracting ADCPM from wireless channel, PCA algorithm is used for preprocessing. The PCA compressed fingerprint will be stored in database. The flowchart of PCA-based fingerprint positioning is shown in Table 2. The simulation will be presented in section 4 .

Table 2. The Flowchart of PCA-based Compressed Fingerprint Localization

\begin{tabular}{|c|c|}
\hline 1 & Offline phase: \\
\hline 1) & $\begin{array}{l}\text { Fingerprint collection: } \\
\text { Divide the target area into grids by definite intervals, collect ADCPM of every grid, use PCA } \\
\text { algorithm to compress ADCPM; }\end{array}$ \\
\hline 2) & $\begin{array}{l}\text { CAOA clustering: } \\
\text { Calculate CAOA of every fingerprint in database, divide the fingerprint with same CAOA into the } \\
\text { same set to form several sets and store them in database; }\end{array}$ \\
\hline 2 & Online phase: \\
\hline 1) & Calculate CAOA of real-time fingerprint of the target terminal; \\
\hline 2) & Find the corresponding set according to CAOA; \\
\hline 3) & $\begin{array}{l}\text { Match the fingerprint of target terminal using WKNN in the set and calculate the position of target } \\
\text { terminal; }\end{array}$ \\
\hline
\end{tabular}

\subsection{The Positioning Method Based on ADF}

Angel delay fingerprint can be extracted from ADCPM easily. Compared with ADCPM, the localization performance based on ADF is much better. The idea of ADF-based localization will be introduced in this section. 


\subsubsection{ADF Extraction}

ADCPM is the mapping of CIR in angle-delay domain, which is a high-dimensional matrix. Figure. 2 shows the distribution of ADCPM channel power. The number of antennas is 64 and the number of cyclic prefix is 754 .

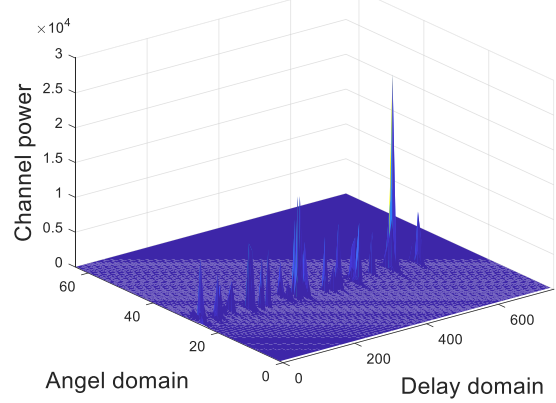

Figure 2. The distribution of ADCPM channel power

The channel power distributes mainly in coordinates $25 \sim 30$ in angle domain. The peak power can be seen in coordinates 400 and 600 in delay domain. When ADCPM is used for fingerprint positioning, the positioning time is long with high computation cost. However, the positioning error is about several meters.

If all the elements in ADCPM are used for positioning, every single element will be calculated by Eq.(1). The elements of fewer channel power which are the majority in ADCPM will be treated the same as the elements of strong channel power which are the minority. Therefore, the positioning error will be affected.

In order to use the positioning information of ADCPM efficiently, the elements which have stronger channel power are selected for positioning, and those elements which have fewer channel power are omitted. The process of ADF extraction from ADCPM is shown in Figure.3.

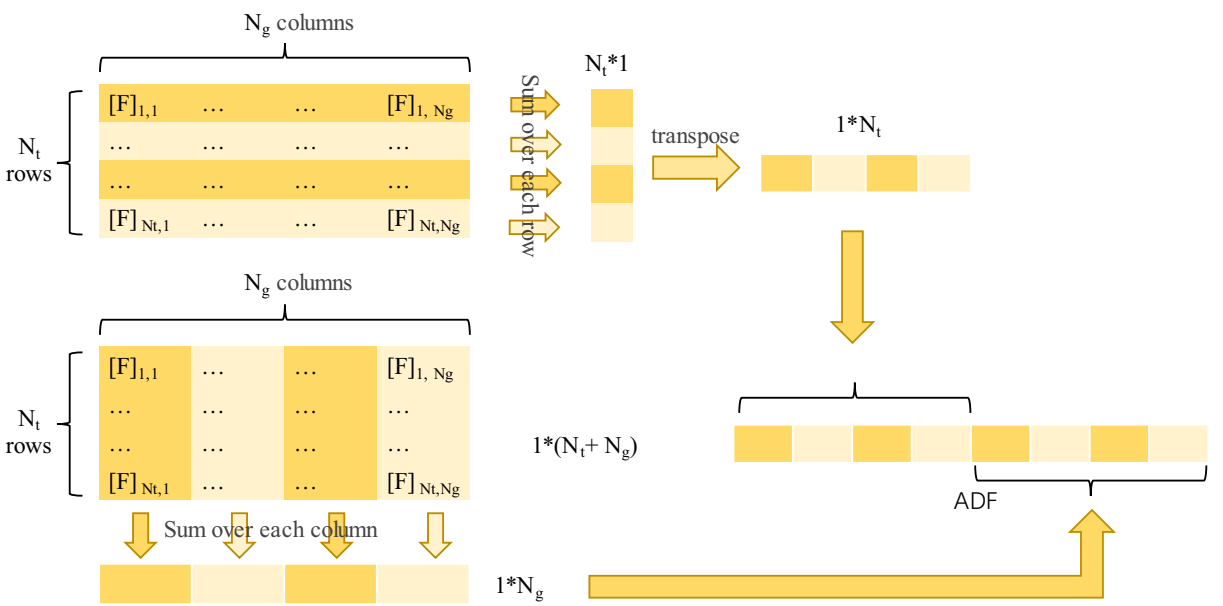

Figure 3. The process of ADF extraction from ADCPM

The extraction of ADCPM is introduced in Section 2 and can be referred to [14] for details. An ADCPM has $N_{t}$ rows and $N_{g}$ columns. Firstly, sum over each row and each column, separately. Then, transpose the $N_{t} * 1$ vector into $1 * N_{t}$ vector. Finally, 
concatenate two vectors into $1 *\left(N_{t}+N_{g}\right)$ which is the final ADF. The process of ADF extraction from ADCPM is easy. The positioning performance of ADF will be introduced in section 4 .

\subsubsection{The Characteristics of $A D F$}

Compared with ADCPM, the data size of ADF is much smaller. For example, if an ADCPM is $64 * 754$, the ADF will be $(64+754) * 1$. The Similarity performance of ADCPM and ADF with Manhattan Distance is shown in Figure.4, which shows that ADF has almost the same similarity performance with ADCPM.

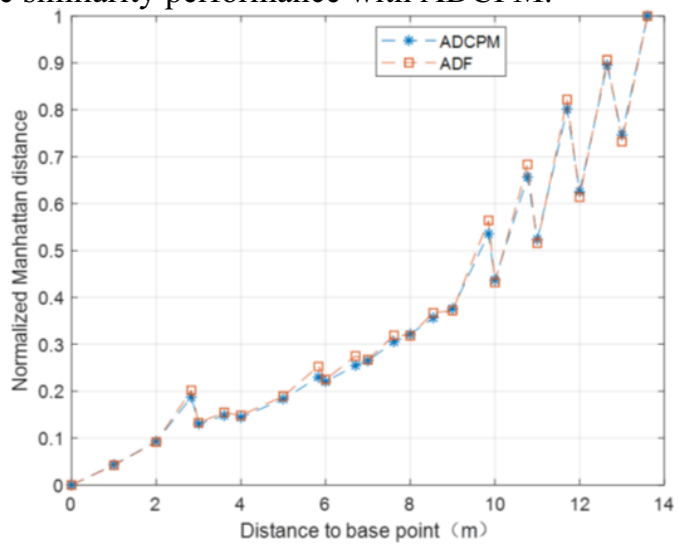

Figure 4. Similarity performance of ADCPM and ADF with Manhattan Distance

\section{Simulation Result}

\subsection{Positioning Time and Storage}

Average positioning time in online phase of three fingerprints is shown in Figure.5. The storage of fingerprint database of three fingerprint is shown in Figure.6. The number of antennas is 64 . The interval of reference points is 1 meter. The density of scatters is $0.02 / \mathrm{m}^{2}$.

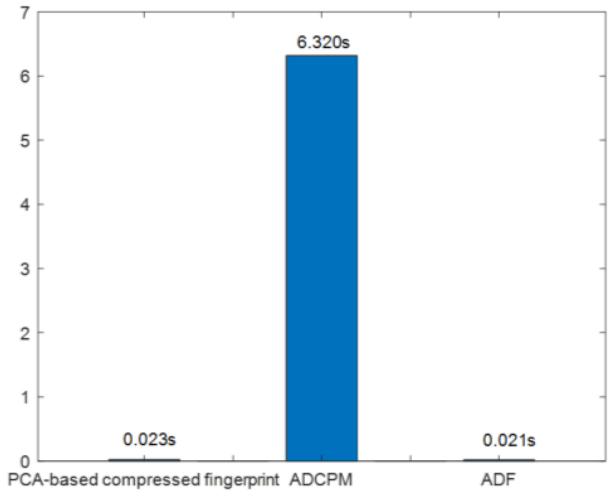

Figure 5. Average positioning time

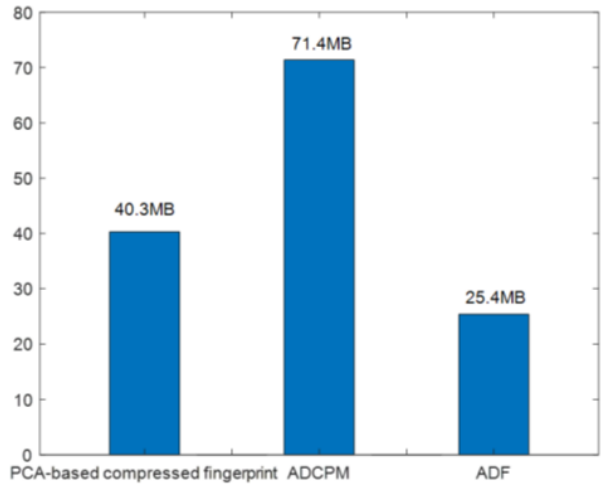

Figure 6. The storage of fingerprint database 
Simulation analysis: The average positioning time of ADCPM is about 6 seconds, which cannot meet real-time requirements of Location-based service (LBS). However, the average positioning time of ADF and PCA-based compressed fingerprint is almost the same, which is about 0.02 seconds. The storage of ADCPM is the largest. However, the storage of ADF is about one-third of ADCPM and the storage of PCA-based compressed fingerprint is about $55 \%$ of ADCPM.

\subsection{Simmulations under Different Wireless Environments}

Wireless environment is very complicated, which has great influence on positioning technology. In this section, we concentrated on positioning performance under different wireless environments, including different number of antennas, bandwidth and density of scatters.

In massive MIMO system, large number of antennas will be used. Average positioning error with different numbers of antennas is shown in Figure.7. The number of antennas is $8,16,32,64,128,256,512$, respectively. The bandwidth of Orthogonal Frequency Division Multiplexing (OFDM) is $80 \mathrm{MHz}$. The density of scatters is $0.02 / \mathrm{m}^{2}$. The interval of reference points is 1 meter.

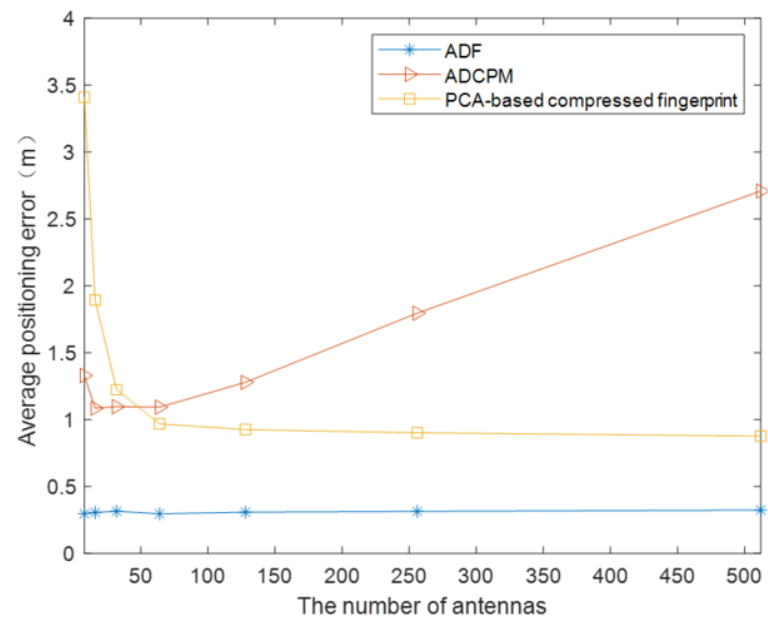

Figure 7. Average positioning error with different number of antennas

Simulation analysis: ADF has the best performance. When the number of antennas changes, average positioning error of $\mathrm{ADF}$ is about 0.3 meter and almost unchanged. However, with the increase of antennas' number, the positioning accuracy of ADCPM becomes worse, while the positioning accuracy of PCA-based compressed fingerprint becomes better. With more antennas, the dimension of ADCPM becomes bigger. The similarity performance of Manhattan distance becomes worse with the increase of weakenergy elements.

Average positioning error with different bandwidth is shown in Figure.8. The number of antennas is 64 . The density of scatters is $0.02 / \mathrm{m}^{2}$. The interval of reference points is 1 meter. 


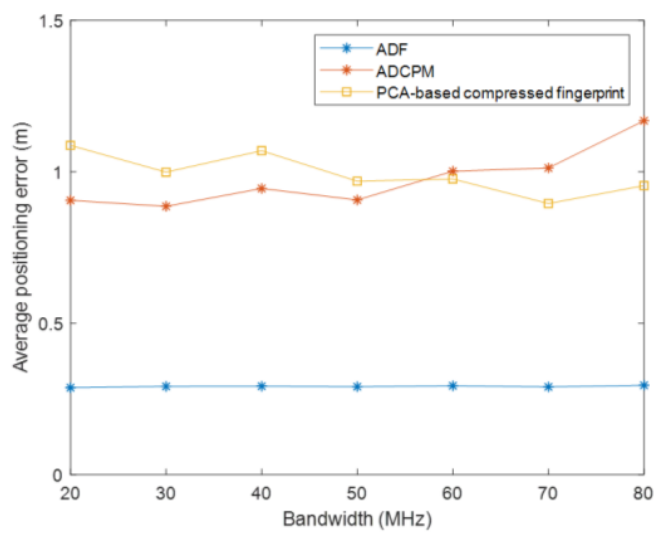

Figure 8. Average positioning error with different bandwidth

Simulation analysis: ADF has the smallest positioning error among these three fingerprints. The average positioning error of ADCPM and PCA-based compressed fingerprint is about 1 meter. With the increase of bandwidth, the positioning accuracy of ADCPM becomes worse and the positioning accuracy of PCA-based compressed fingerprint becomes better. This is the same situation as different number of antennas. With bigger bandwidth, elements of weak channel power take up a larger proportion of ADCPM. Therefore, the similarity performance of Manhattan distance becomes worse.

Finally, we investigate average positioning error with different density of scatters, and the simulation results is shown in Figure.9. The bandwidth of OFDM is $80 \mathrm{MHz}$. The number of antennas is 64 . The interval of reference points is 1 meter. The density of scatter changes from $0.005 / \mathrm{m}^{2}$ to $0.2 / \mathrm{m}^{2}$.

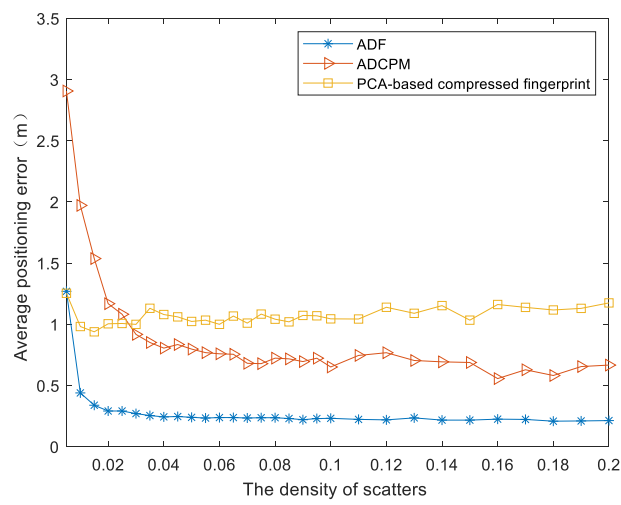

Figure 9. Average positioning error with different density of scatters

Simulation analysis: With the increase of the density of scatters, more CSI information will be provided due to multipath effect. Hence, the positioning error of three fingerprints becomes smaller. Within these three fingerprint, ADF has the best positioning accuracy. When the density of scatters is smaller than $0.03 / \mathrm{m}^{2}$, PCA-based compressed fingerprint is better than ADCPM. When the density of scatters is bigger than $0.03 / \mathrm{m}^{2}$, PCA-based compressed fingerprint performs worse. With more multipath information, ADCPM has better positioning accuracy, but in PCA algorithm, the compressed fingerprint remains in a low-dimensional space and the loss of effective positioning information is more. 


\section{Conclusion}

In this paper, we have investigated high-performance positioning algorithms based on massive MIMO system. Firstly, the channel model is introduced and the extraction of ADCPM fingerprint is introduced. Then, two kinds of new fingerprint are proposed. One is PCA-based compressed fingerprint, which use PCA algorithm to extract efficient information from ADCPM. Simulation results show that PCA-based compressed fingerprint positioning can reduce average positioning time and the storage overhead. However, the positioning performance of ADF is much better. The average positioning error is about 0.3 meter, which is one third of ADCPM. What's more, the positioning time is about tens of milliseconds and the data size of fingerprint database is about one third of ADCPM. Finally, numerical results demonstrate the performance of those two new fingerprints.

\section{References}

[1] G. Wang, H. Chen, Y. Li and N. Ansari, "NLOS Error Mitigation for TOA-Based Localization via Convex Relaxation," in IEEE Transactions on Wireless Communications, vol. 13, no. 8, pp. 4119-4131, Aug. 2014, doi: 10.1109/TWC.2014.2314640.

[2] X. Guo, Y. Huang, B. Li and L. Chu, "DOA estimation of mixed circular and non-circular signals using uniform circular array," 2014 7th International Congress on Image and Signal Processing, Dalian, 2014, pp. 1043-1047, doi: 10.1109/CISP.2014.7003933.

[3] G. Wang, Y. Li and N. Ansari, "A Semidefinite Relaxation Method for Source Localization Using TDOA and FDOA Measurements," in IEEE Transactions on Vehicular Technology, vol. 62, no. 2, pp. 853-862, Feb. 2013, doi: 10.1109/TVT.2012.2225074.

[4] Honkavirta, Ville, et al. "A comparative survey of WLAN location fingerprinting methods." Positioning, Navigation and Communication, 2009. WPNC 2009. 6th Workshop on IEEE, 2009.

[5] Zhang, Lei, et al. "A novel fingerprinting using channel state information with MIMO-OFDM." Cluster Computing (2017).

[6] X. Wang, L. Gao, S. Mao and S. Pandey, "DeepFi: Deep learning for indoor fingerprinting using channel state information," 2015 IEEE Wireless Communications and Networking Conference (WCNC), New Orleans, LA, 2015, pp. 1666-1671, doi: 10.1109/WCNC.2015.7127718.

[7] Y. Lin, P. Tseng, Y. Chan and G. Wu, "Super-Resolution-Aided Positioning Fingerprinting Based on Channel Impulse Response Measurement," 2017 IEEE Wireless Communications and Networking Conference (WCNC), San Francisco, CA, 2017, pp. 1-6, doi: 10.1109/WCNC.2017.7925866.

[8] N. Garcia, H. Wymeersch, E. G. Larsson, A. M. Haimovich and M. Coulon, "Direct Localization for Massive MIMO," in IEEE Transactions on Signal Processing, vol. 65, no. 10, pp. 2475-2487, 15 May15, 2017, doi: 10.1109/TSP.2017.2666779.

[9] H. Xie et al., "Accelerating Crowdsourcing Based Indoor Localization Using CSI," 2015 IEEE 21st International Conference on Parallel and Distributed Systems (ICPADS), Melbourne, VIC, 2015, pp. 274-281, doi: 10.1109/ICPADS.2015.42.

[10] T. L. Marzetta, "Noncooperative Cellular Wireless with Unlimited Numbers of Base Station Antennas," in IEEE Transactions on Wireless Communications, vol. 9, no. 11, pp. 3590-3600, November 2010, doi: 10.1109/TWC.2010.092810.091092.

[11] Larsson E G, Edfors O, Tufvesson F, et al. Massive MIMO for next generation wireless systems[J]. IEEE Communications Magazine, 2014, 52(2):186-195.

[12] Alamu O, Iyaomolere B, et al. An overview of massive MIMO localization techniques in wireless cellular networks: Recent advances and outlook[J]. Ad Hoc Networks, 2021, 111:102353.

[13] Sun X, Gao X, et al. Single-Site Localization Based on a New Type of Fingerprint for Massive MIMOOFDM Systems[J]. IEEE Transactions on Vehicular Technology, 2018, 67(7): 6134-6145.

[14] Sun X, Wu C, et al. Fingerprint-based Localization for Massive MIMO-OFDM System with Deep Convolutional Neural Networks[J]. IEEE Transactions on Vehicular Technology, 2019, PP (99):1-1. 